type 2 (HSV-2) among female sex workers (FSWs) in the Middle East and North Africa (MENA), a neglected research area.

Methods Literature was systematically reviewed, with findings reported following PRISMA guidelines. Pooled prevalences of current and/or lifetime infection for each STI were estimated using random-effects meta-analyses. Sources of between-study heterogeneity were determined through meta-regressions.

Results One T. pallidum incidence study and 144 STI prevalence studies were identified for 45,812 FSWs in 13 MENA countries. Pooled prevalence of current infection was $12.7 \%$ (95\% confidence interval-CI: $8.5-17.7 \%$ ) for $T$. pallidum, 14.4\% (95\% CI: 8.2-22.0\%) for C. trachomatis, $5.7 \%$ (95\% CI: $3.5-8.4 \%$ ) for N. gonorrhoeae, and $7.1 \%$ (95\% CI: $4.3-10.5 \%$ ) for T. vaginalis. Pooled prevalence of lifetime infection was $12.8 \%$ (95\% CI: 9.4-16.6\%) for $T$. pallidum, 80.3\% (95\% CI: 53.2-97.6\%) for C. trachomatis, and $23.7 \%$ (95\% CI: 10.2-40.4\%) for HSV-2. The multivariable meta-regression for $T$. pallidum prevalence demonstrated strong subregional differences, with the Horn of Africa and North Africa showing, respectively, six-fold (adjusted odds ratio (AOR): 6.4; 95\% CI: 2.5-16.8) and five-fold (AOR: 5.0; 95\% CI: 2.4-10.6) higher odds for prevalence than Eastern MENA. There was also strong evidence for a declining $T$. pallidum prevalence at a rate of 7\% per year (AOR: 0.93; 95\% CI: 0.88-0.98). Study-specific factors including diagnostic method, sample size, sampling methodology, and response rate, were not associated with syphilis prevalence.

Conclusion STI infection levels among FSWs in MENA are considerable, supporting a key role for commercial heterosexual sex networks in transmission dynamics, and highlighting the health needs of this neglected and vulnerable population. Syphilis prevalence in FSWs appears to be declining for at least three decades. Gaps in evidence persist for multiple countries.

Disclosure No significant relationships.

\section{P696 HIV AMONG FEMALE SEX WORKERS AND CLIENTS IN THE MIDDLE EAST AND NORTH AFRICA: SUBREGIONAL DIFFERENCES AND EPIDEMIC POTENTIAL}

${ }^{1}$ Hiam Chemaitelly ${ }^{*},{ }^{2}$ Helen Weiss, ${ }^{3}$ Clara Calvert, ${ }^{1}$ Manale Harfouche, ${ }^{4}$ Laith Abu-Raddad. ${ }^{1}$ Weill Cornell Medicine-Qatar, Infectious Disease Epidemiology Group, Doha, Qatar; ${ }^{2}$ London School of Hygiene and Tropical Medicine, MRC Tropical Epidemiology Group, London, UK; ${ }^{3}$ London School of Hygiene and Tropical Medicine, Department of Infectious Disease Epidemiology, London, UK; ${ }^{4}$ Weill Cornell Medicine-Qatar, Doha, Qatar

\subsection{6/sextrans-2019-sti.762}

Background This study addresses the gap in our understanding of HIV epidemiology among female sex workers (FSWs) and clients in the Middle East and North Africa (MENA) region.

Methods An exhaustive systematic review of population-size estimation and of HIV prevalence studies was conducted. Findings were reported following PRISMA guidelines. The pooled mean HIV prevalence was estimated using random-effects metaanalyses. Associations with prevalence, sources of heterogeneity, and temporal trends were investigated using meta-regressions.

Results We identified 270 size-estimation studies in FSWs and 42 in clients, as well as 485 HIV prevalence studies on
287,719 FSWs, and 69 on 29,531 clients/proxy populations (male sexually transmitted infections clinic attendees). The median proportion of reproductive-age women reporting current $/$ recent sex work was $0.7 \%$ (range $=0.2-2.4 \%$ ), and of men reporting currently/recently buying sex was $5.7 \%$ (range $=0.3-13.8 \%$ ). HIV prevalence ranged from $0-70 \%$ in FSWs (median $=0.1 \%$ ), and $0-34.6 \%$ in clients (median $=0.4 \%$ ). The regional pooled mean HIV prevalence was $1.4 \%(95 \% \quad \mathrm{CI}=1.1-1.8 \%)$ in $\mathrm{FSWs}$ and $0.4 \%(95 \%$ $\mathrm{CI}=0.1-0.7 \%)$ in clients. Country-specific pooled HIV prevalence in FSWs was $<1 \%$ in most countries, $1-5 \%$ in North Africa and Somalia, $17.3 \%$ in South Sudan, and 17.9\% in Djibouti. Meta-regressions identified strong subregional variations in prevalence, where compared to Eastern MENA, the adjusted odds ratios (AORs) ranged from 0.2 (95\% CI=0.10.4) in Fertile Crescent to 46.3 (95\% CI=25.9-82.6) in Horn of Africa. There was also strong evidence for increasing prevalence post-2003, at a rate of $14 \%$ per year (AOR $=1.14,95 \%$ $\mathrm{CI}=1.08-1.20)$.

Conclusion HIV epidemics among FSWs are emerging in MENA, with some already in an established phase, though still some countries have limited epidemic dynamics. The epidemic has been growing for over a decade, with strong regionalization and heterogeneity.

Disclosure No significant relationships.

\section{P697 FEASIBILITY OF HPV SELF-COLLECTION FOR CERVIX SCREENING IN UNDER-SCREENED STREET ENTRENCHED WOMEN}

${ }^{1}$ Sheona Mitchell-Foster*, ${ }^{2}$ C.Sarai Racey, ${ }^{3}$ Tracey Day, ${ }^{1}$ Celena Falkner, ${ }^{4}$ Laurie Smith, ${ }^{2}$ Heather Pedersen, ${ }^{5}$ Tracy Chan, ${ }^{5}$ Darrel Cook, ${ }^{6}$ Kate Shannon, ${ }^{4}$ Marette Lee, ${ }^{2}$ Deborah Money, ${ }^{3}$ Sandra Allison, ${ }^{6}$ Jill Chettiar, ${ }^{2}$ Gina Ogilvie. ${ }^{1}$ University of Northern British Columbia, Prince George, Canada; ' University of British Columbia, Vancouver, Canada; ${ }^{3}$ Northern Health Authority, Prince George, Canada; ${ }^{4} B C$ Cancer, Vancouver, Canada; ${ }^{5} B C$ Centre for Disease Control, Vancouver, Canada; ${ }^{6}$ University of British Columbia, Centre for Gender and Sexual Health Equity, Vancouver, Canada

\subsection{6/sextrans-2019-sti.763}

Background HPV self-collection is a promising approach to improve uptake of cervical screening in under-screened women. The aim of this feasibility study was to measure uptake of HPV self-collection, HPV positivity, and screening history of street entrenched women in a rural region centre.

Methods Women 30-69 years of age, attending drop-in community-based primary care and integrative reproductive health clinics in Northern British Columbia (BC), Canada and selfreported not having received cervix screening in the last 3 years, were offered self-collection for HPV testing. A convenience sample of all comers was administered a questionnaire and underwent a medical chart review, including the provincial cervix screening registry. Demographics, HIV status, and cervix screening history were collected. All women who tested HPV16/18 positive were referred for colposcopy.

Results A total of 66 eligible women were analyzed (mean age 43.3 years), with population saturation reached after 3 months recruitment. An additional 11 women were deemed ineligible due to age or prior hysterectomy. $83 \%$ self-reported as Indigenous. Based on the provincial cervix screening registry, $48 \%$ of women were up-to-date on cervix screening based on 
triennial screening guidelines. All women undertook self-collection and the majority of women reported high perceived acceptability, safety, and accuracy of HPV self-collection. HPV $16 / 18$ positivity was $7.6 \%$, with $40 \%$ co-infected with HIV. Overall HIV prevalence was $16.4 \%$, however, over $25 \%$ of women had unknown HIV status based on medical chart review.

Conclusion HPV self-collection was highly acceptable as part of community-based integrative reproductive health services. Despite being a traditionally underserved population, and women self-reporting being overdue for screening, over half the women were up to date on cervix screening, albeit regular screening was lacking for many. The findings from this feasibility study will inform future implementation of HPV self-collection to improve and maintain regular cervix screening services in street entrenched women.

Disclosure No significant relationships.

\section{P698 COMPREHENSIVE HEALTHCARE INTERVENTION FOR FEMALE SEX WORKERS IN ETHIOPIAN STI CLINICS: EXPERIENCE FROM MEKELLE UNIVERSITY}

\begin{abstract}
${ }^{1}$ Tesfay Gebrehiwot*, ${ }^{2}$ Zerihun Abebe, ${ }^{3}$ Dejen Yemane, ${ }^{3}$ Solomon Gebremariam, ${ }^{4}$ Loko Bongassie. 'Mekelle University, School of Public Health, Department of Health Systems, Mekelle, Ethiopia; ${ }^{2}$ Saint Paulos Millenium Medical College, Addis Ababa, Ethiopia; ${ }^{3}$ Mekelle University, Mekelle, Ethiopia; ${ }^{4}$ Ethiopian Pharmacuetical Fund Supply Agency, Mekelle, Ethiopia
\end{abstract}

\subsection{6/sextrans-2019-sti.764}

Background Evidence suggests that the presence of untreated sexually transmitted infections (STIs) increases the chance of HIV transmission during unprotected sex. In the Ethiopian context, many female sex workers live in poor conditions in rented slums and are not typically known or recognised by local authorities, making them unable to access health facilities. Methods Data were obtained from a register of female sex workers recorded for purpose of service provision at confidential STI clinics in Mekelle and Adigrat, Ethiopia, from May 2010 to August 2015 and from May 2011 to August 2015, respectively. A simple descriptive analysis of services delivered to patients was performed.

Results Among the 6288 patients included in this study, the prevalence of STIs was 23.4\%. Of these, $12.9 \%(814 / 6288)$ of patients presented with vaginal discharge, $7.9 \%(490 / 6288)$ with genital ulcers and $2.3 \%(158 / 6288)$ with lower abdominal pain (as per the WHO syndromic approach to STIs). Moreover, 180 cases of genital ulcer were tested for syphilis with the VDRL test; 36 (20\%) tested positive for active infection. The HIV prevalence declined from $10 \%$ in 2010 to $1.1 \%$ in 2015 . The frequency of STIs amongst repeat patients was considerably lower than that in new presentations.

Conclusions HIV prevalence declined from 10\% in 2010 to $1.1 \%$ in 2015 . Compared to new cases, the frequency of STIs among repeat clients was extremely low, suggesting that the cumulative effect of peer promotion and preventative sexual health education is effective in reducing the rates of STIs among vulnerable populations. Clinic and workplace geography, hours of clinic operation, confidentiality and peer outreach are important factors in the prevention and control of STI/HIV infection in key sex worker populations. A comprehensive clinic intervention enhances early diagnosis and treatment of STIs and increases the proportion of sex workers accessing HIV treatment services.
Disclosure No significant relationships.

\section{P699 ROLE OF MANAGEMENT IN ENHANCING EFFICIENCY OF FEMALE SEX WORKERS HIV PROGRAMS IN NIGERIA}

Idoteyin Ezirim*, Francis Agbo. National Agency for the Control of AIDS, Research, Monitoring and Evaluation, Abuja, Nigeria

\subsection{6/sextrans-2019-sti.765}

Background HIV is high among female sex workers (FSW). Community-based organizations (CBOs) staff and volunteers, deliver HIV services to FSWs. One overlooked avenue for enhancing efficiency in delivery of services is good personnel management. This study was done to understand challenges faced by $\mathrm{CBO}$ staff during service delivery and management practices hindering their output.

Methods Qualitative methods were used in this study. Staffs and volunteers from 9 CBOs in three states, Abuja, Lagos and Nasarrawa participated in the study. States were selected using convenience sampling. Journals were distributed to $\mathrm{CBO}$ staff to document their daily challenges for 3-6 weeks. After 6 weeks, we collected 23 journals, read entries and conducted 6 non-participant observations and 31 key informant interviews. Information from the completed journals, interviews and observations, were grouped into themes to reveal insights. Using these insights, we conducted brainstorming sessions with CBOs to generate problem-solving ideas. The most relevant ideas were tested in focus groups.

Results The study revealed that CBOs provide HIV services as agreed with donors. They did not solicit and implement feedback from volunteers. Instituting feedback mechanisms will make CBO personnel feel relevant and serve as an avenue for harvesting solutions to challenges in service delivery. The study showed that FSW peer session schedules change constantly and volunteers find it difficult to communicate changes to CBO staff resulting in poor supervision. Poor supervision affects the quality of services volunteers provide. Therefore ensuring communication within $\mathrm{CBO}$ personnel, will improve coordination of activities and quality of HIV services delivered. Finally, while CBOs have systems in place to reward performance, these systems were underutilized and not incentivized. If reward systems for best practices are rejuvenated, it will motivate personnel.

Conclusion Good personnel management can enhance FSW HIV programs. Feedback mechanisms, communication and reward systems are tools that will ensure efficient delivery HIV prevention services.

Disclosure No significant relationships.

\section{P700 UNINTENDED PREGNANCY AMONG FEMALE SEX WORKERS IN MEKELLE CITY, NORTHERN ETHIOPIA: A CROSS-SECTIONAL STUDY}

${ }^{1}$ Tesfay Gebrehiwot*, ${ }^{2}$ Rishan Weldegebriel, ${ }^{3}$ Mussie Alemayehu. 'Mekelle University, School of Public Health, Department of Health Systems, Mekelle, Ethiopia; ${ }^{2}$ Mekelle University, City, Ethiopia; ${ }^{3}$ Mekelle University, Mekelle, Ethiopia

\subsection{6/sextrans-2019-sti.766}

Background Unintended pregnancy is a significant public health concern in the world. Particularly, female sex workers are exposed to the risk of unintended pregnancy, abortion and 